\title{
A new method of extraction of a $p$ - $n$ diode series resistance from $I-V$ characteristics and its application to analysis of low-temperature conduction of the diode base
}

\author{
V.L. Borblik*, Yu.M. Shwarts, M.M. Shwarts \\ V. Lashkaryov Institute of Semiconductor Physics, National Academy of Sciences of Ukraine, \\ 41, prospect Nauky, 03028 Kyiv, Ukraine \\ Phone: +38(044)525-6292, fax: +38(044)525-7463 \\ *E-mail: borblik@isp.kiev.ua
}

\begin{abstract}
A new analytical method of extraction of a diode series resistance from current-voltage characteristics is proposed which takes into account dependence of the series resistance on voltage (or current). The method supposes a presence of linear section in the diode current-voltage characteristic plotted in semi-logarithmic scale. This method is applied here to experimental data for silicon diode in which series resistance is caused by freezing-out free current carriers into impurities at cryogenic temperatures. Character of dependence of the base resistance on electric field in the base layer determined in such way confirms hopping nature of silicon conduction under these conditions.
\end{abstract}

Keywords: junction diode, series resistance, silicon, metal-insulator transition, low temperature, hopping conduction.

Manuscript received 21.08.09; accepted for publication 10.09.09; published online 30.10.09.

\section{Introduction}

There exist many methods of series resistance extraction from current-voltage characteristics (CVC) of Schottky or $p$ - $n$ junction diode. Their description and comparison with each other one can find in papers [1-8] and references therein. But any of these methods is not applicable in the case if the series resistance varies with voltage (or current). Only paper [9] takes into account a base resistance modulation in Schottky diode but the method used in [9] is lengthy iteration procedure relative to the transcendental equation. We propose in this paper a simple analytical method suitable for $p$ - $n$ junction diode where the series resistance appears as a result of freezing-out current carriers into impurities at cryogenic temperatures.

\section{Experimental data}

We investigated silicon $n^{+}-p$ diodes with base doping level (by boron) which does not reach somewhat the critical concentration $N_{c}$ for insulator-metal transition ( $N_{c}$ for $p$-Si is $4 \cdot 10^{18} \mathrm{~cm}^{-3}[10]$ ). Temperature family of
CVC for one of these diodes (GPI-1025) at cryogenic temperatures is represented in Fig. 1. It is worthwhile to compare it (in the same temperature range and in the same scale) with CVC of the silicon $n^{++}-p^{+}$diode (DT67) whose base is doped to the level which is somewhat higher than the critical concentration $N_{c}$ (Fig. 2). In this case the diode series resistance is absent (negligibly small). Conduction of such the diode as it has been shown by us in [11] is realized by tunnel-recombination mechanism which is connected with localized electron states in the forbidden band [12]. The CVC are described by the dependence $I \propto \exp \left(U / U_{t}\right)$ where $U_{t}^{-1}$ is temperature-independent parameter related to tunneling through the barrier (equal to $80 \mathrm{~V}^{-1}$ in the given case).

It is obvious of Figs 1 and 2 that at the base doping level which is somewhat lower than critical one, mechanism of conduction through the junction barrier remains practically the same; simply the series resistance $R_{s}$ superimposes on it. Therefore CVC take the form

$$
I=I_{0} \exp \left(\frac{U-I R_{S}}{U_{t}}\right),
$$




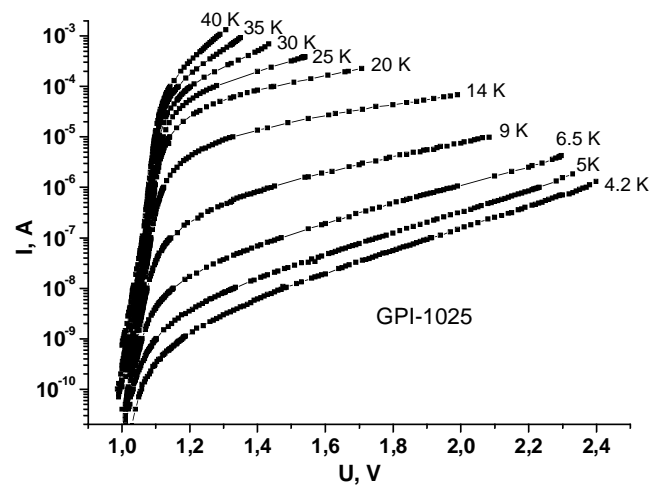

Fig. 1. Current-voltage characteristics of investigated silicon diode in cryogenic temperature region.

where, however, parameter $U_{t}^{-1}$ (well defined in view of presence of linear sections in CVC) acquires weak temperature dependence remaining the same by the order of magnitude (from 70 to $100 \mathrm{~V}^{-1}$ ). Any of the methods of extracting $R_{s}$ which considers $R_{s}$ as constant does not give a result here. Therefore necessity of new method arises which would take into account dependence of $R_{s}$ on $U$ (or $I$ ).

\section{Differential method of extracting diode series resistance depending on voltage (or current)}

For more generality, let us consider CVC in the form

$I=I_{0} \exp \left[\alpha\left(U-I R_{S}\right)\right]$,

where $\quad \alpha=q / n k T$ for classical current-conducting mechanisms (diffusion or generation-recombination; then $I_{0}$ is the saturation current, $q$ is an electron charge, $k$ is the Boltzmann constant, $T$ is temperature, $n$ is the ideality factor) and $\alpha=U_{t}^{-1}$ in our case. Representing equation (2) in the form

$\ln \left(\frac{I}{I_{0}}\right)=\alpha\left(U-I R_{S}\right)$

and considering $\alpha=$ const differentiate (3) with respect to $U$ (traditional approach)

$\frac{d \ln I}{d U}=\alpha\left(1-\frac{d I}{d U} R_{S}-I \frac{d R_{S}}{d U}\right)$,

then with respect to $I$

$\frac{1}{I}=\alpha\left(\frac{d U}{d I}-R_{S}-I \frac{d R_{S}}{d I}\right)$,

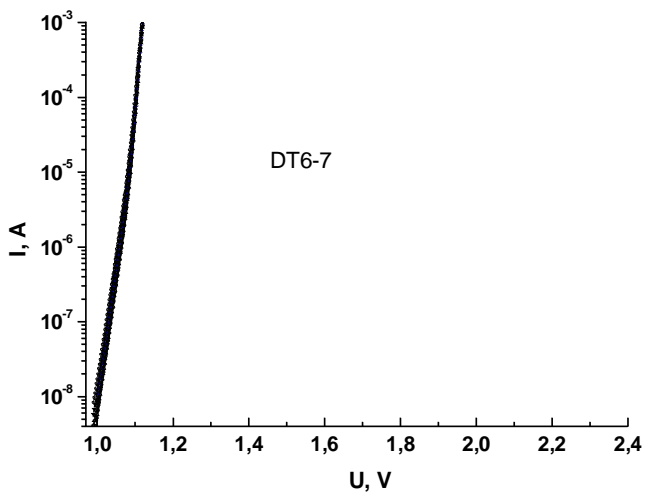

Fig. 2. Current-voltage characteristics of the silicon diode with high doping level of the base in the same temperature range as in Fig. 1.

and, finally, with respect to $\ln I$ (as in paper [1])

$1=\alpha\left(\frac{d U}{d \ln I}-I R_{s}-I \frac{d R_{S}}{d \ln I}\right)$.

Equations (4) through (6) are linear differential equations for $R_{s}$. Any of them results in solution

$R_{S}=\frac{1}{I}\left[U-U_{\min }-\frac{1}{\alpha}\left(\ln I-\ln I_{\min }\right)\right]$,

where $U_{\min }$ and $I_{\min }$ are coordinates of any point (but best of the lowest one) of the linear section of CVC (see Fig. 3). In this point the boundary condition is set: $R_{S}\left(I_{\min }, U_{\min }\right)=0$.

From (7) the expression for the voltage drop across the diode base follows as well

$U_{\text {base }}=U-U_{\min }-\frac{1}{\alpha}\left(\ln I-\ln I_{\min }\right)$.

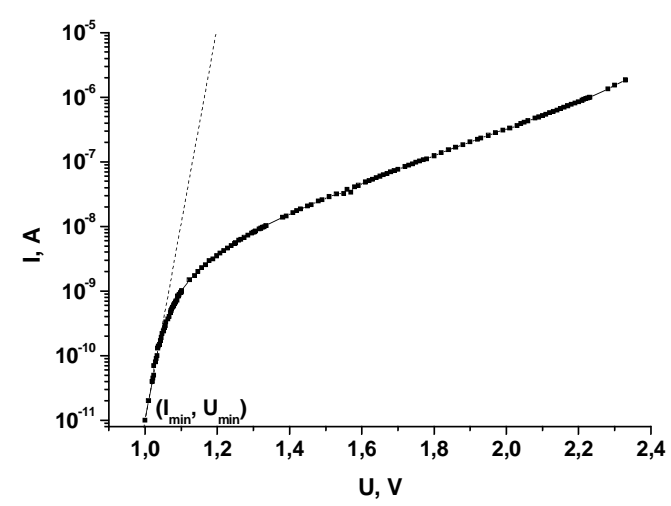

Fig. 3. Example of processing the typical current-voltage characteristic. 


\section{Application of the theory to the experimental data}

Shown in Fig. 4 are the values of $R_{s}$ calculated by formula (7) plotted against $U_{\text {base }}$ calculated by formula (8) in the temperature range $4.2-14 \mathrm{~K}$. And similar calculation results for the temperature range $15-35 \mathrm{~K}$ are presented in Fig. 5. Note that at more low temperatures (Fig. 4), the diode base resistance at first increases and then exhibits exponential drop with the base voltage. At higher temperatures (Fig. 5) the base resistance decreases with the base voltage (after passing the maximum) non-exponentially.

Though there exist the papers (see, for example, [13]) which predict increase of a diode series resistance at the expense of minority carrier injection, in our case reason is likely different. It appears to be connected simply with gradual transition from the situation where $p$ - $n$ junction resistance is considerably greater than $R_{S}$ (i.e. $\left.R_{s} \approx 0\right)$, through the situation when $p-n$ junction resistance becomes comparable with $R_{s}$, to the situation where $R_{s}$ dominates in total diode resistance (maximum point). Subsequent decrease of $R_{s}$ is its intrinsic variation with base voltage because of the electric field growth.

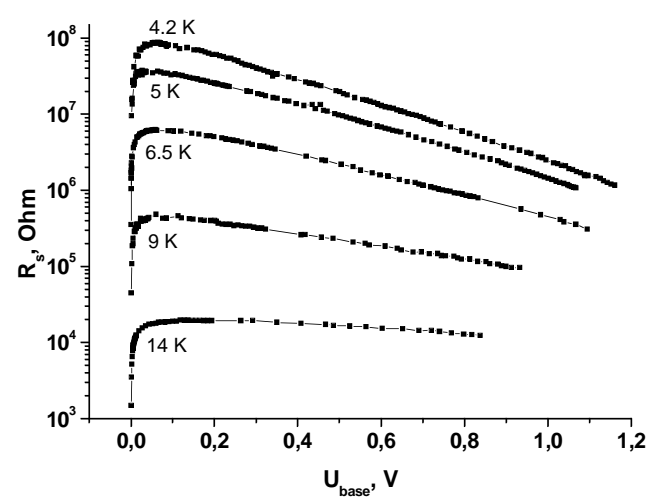

Fig. 4. Dependence of calculated diode series resistance on the base voltage in temperature range $4.2-14 \mathrm{~K}$.

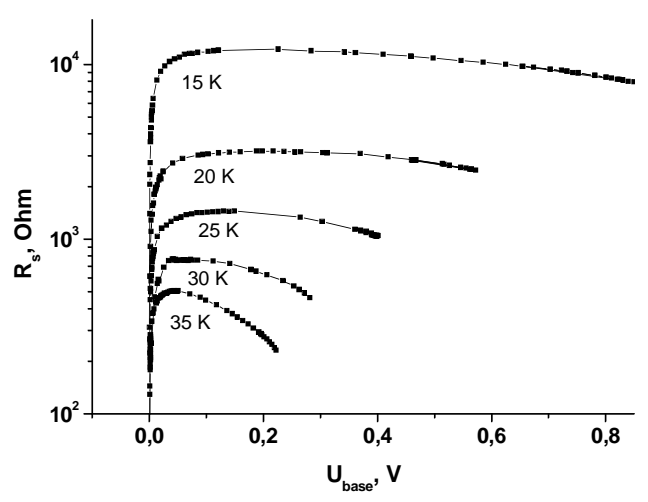

Fig. 5. Dependence of calculated diode series resistance on the base voltage in temperature range $15-35 \mathrm{~K}$.

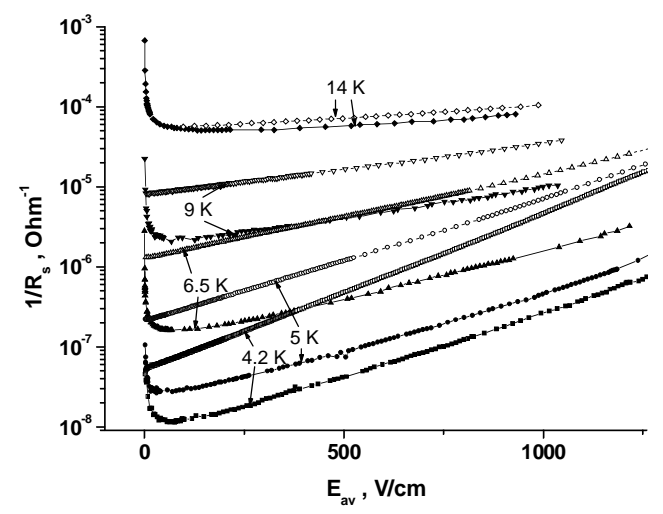

Fig. 6. Comparison of electric-field dependences of the diode (closed symbols) and the testing resistor (open symbols) conductivity.

It is worthwhile to compare dependence of the base conductivity $R_{s}^{-1}$ (when $R_{s}$ decreases with the base voltage) on average electric field $E_{a v}$ in the base layer with the field dependence of conductivity for silicon testing resistor whose electric properties (conductivity type and doping) are equivalent to the investigated diode base layer. Such measurements have been carried out by us in paper [14] and they have established hopping nature of the testing resistor conductivity at low temperatures.

Fig. 6 shows the results of such comparison: closed symbols - for the diode base conductivity, open symbols - for the testing resistor one. Both dependences (for each temperature) prove to be rather similar in their exponential portions. This gives evidence that in the diode, under injection conditions, conductivity of silicon doped below critical concentration keeps, at low temperatures, its hopping character.

\section{Conclusion}

So, for the first time, there has been proposed an analytical method of extraction of a diode series resistance from the diode CVC taking into account dependence of the series resistance on voltage drop across the diode or across the diode base. Being applied to experimental data for silicon diode under conditions of freezing-out free carriers into impurities the method has allowed us to establish hopping nature of the diode base conductivity.

\section{References}

1. S.K. Cheung and N.W. Cheung, Extraction of Schottky diode parameters from forward currentvoltage characteristics // Appl. Phys. Lett. 49 (2), p. 85-87 (1986). 
2. T.C. Lee, S. Fung, C.D. Beling, and H.L. Au, A systematic approach to the measurement of ideality factor, series resistance, and barrier height for Schottky diodes // J. Appl. Phys. 72 (10), p. 47394742 (1992).

3. V. Aubry and F. Meyer, Schottky diodes with high series resistance: Limitations of forward $I-V$ methods // J. Appl. Phys. 76 (12), p. 7973-7984 (1994).

4. M. Lyakas, R. Zaharia, and M. Eizenberg, Analysis of nonideal Schottky and $p$ - $n$ junction diodes extraction of parameters from $I-V$ plots // J. Appl. Phys. 78 (9), p. 5481-5489 (1995).

5. V. Mikhelashvili, G. Eisenstein, V. Garber, S. Fainleib, G. Bahir, D. Ritter, M. Orenstein, and A. Peer, On the extraction of linear and nonlinear physical parameters in nonideal diodes // J. Appl. Phys. 85 (9), p. 6873-6883 (1999).

6. A. Kaminski, J.J. Marchand, and A. Laugier, I-V methods to extract junction parameters with special emphasis on low series resistance // Solid-State Electron. 43 (4), p. 741-745 (1999).

7. C.-C. Liu, C.-Y. Chen, C.-Y. Weng, C.-C. Wang, F.-L. Jenq, P.-J. Cheng, Y.-H. Wang, and M.-P. Houng, Physical parameters extraction from current-voltage characteristic for diodes using multiple nonlinear regression analysis // Solid-State Electron. 52 (6), p. 839-843 (2008).
8. W. Jung and M. Guziewicz, Schottky diode parameters extraction using Lambert $\mathrm{W}$ function // Mater. Sci. Eng. B (2009), doi:10.1016 / j.mseb.2009.02.013.

9. A.I. Prokopyev and S.A. Mesheryakov, Fast extraction of static parameters of Schottky diodes from forward $I-V$ characteristic // Measurement 37 (2), p. $149-155$ (2005).

10. P. Dai, Y. Zhang, and P.M. Sarachik, Critical conductivity exponent for Si:B // Phys. Rev. Lett. 66 (4), p. 1914-1917 (1991).

11. V.L. Borblik, Yu.M. Shwarts and M.M. Shwarts, Revealing the hopping mechanism of conduction in heavily doped silicon diodes // Semiconductor Physics, Quantum Electronics \& Optoelectronics 8 (2), p. 41-44 (2005).

12. J.A. Del Alamo and R.M. Swanson, Forward-bias tunneling: a limitation to bipolar device scaling // IEEE Electron. Device Lett. EDL-7 (11), p. 629631 (1986).

13. J.-C. Manifacier and H.K. Henisch, Minoritycarrier injection into semiconductors // Phys. Rev.B 17 (6), p. 2640-2647 (1978).

14. Yu.M. Shwarts, A.V. Kondrachuk, M.M. Shwarts, and L.I. Shpinar, Non-ohmic Mott conductivity and thermometric characteristics of heavily doped silicon structures // Semiconductor Physics, Quantum Electronics \& Optoelectronics 3 (3), p. 400-405 (2000). 